\title{
Spatio-temporal elements of articulation work in the achievement of repeat prescribing safety in UK general practice
}

Citation for published version (APA):

Grant, S., Mesman, J., \& Guthrie, B. (2016). Spatio-temporal elements of articulation work in the achievement of repeat prescribing safety in UK general practice. In D. Allen, J. Braithwaite, J. Sandall, \& J. Waring (Eds.), The Sociology of Healthcare Safety and Quality (pp. 121-139). Wiley-Blackwell. Sociology of Health and IIIness Monograph Series https://doi.org/10.1002/9781119276371.ch8

Document status and date:

Published: 01/01/2016

DOI:

10.1002/9781119276371.ch8

Document Version:

Publisher's PDF, also known as Version of record

Document license:

Taverne

Please check the document version of this publication:

- A submitted manuscript is the version of the article upon submission and before peer-review. There can be important differences between the submitted version and the official published version of record.

People interested in the research are advised to contact the author for the final version of the publication, or visit the DOI to the publisher's website.

- The final author version and the galley proof are versions of the publication after peer review.

- The final published version features the final layout of the paper including the volume, issue and page numbers.

Link to publication

\footnotetext{
General rights rights.

- You may freely distribute the URL identifying the publication in the public portal. please follow below link for the End User Agreement:

www.umlib.nl/taverne-license

Take down policy

If you believe that this document breaches copyright please contact us at:

repository@maastrichtuniversity.nl

providing details and we will investigate your claim.
}

Copyright and moral rights for the publications made accessible in the public portal are retained by the authors and/or other copyright owners and it is a condition of accessing publications that users recognise and abide by the legal requirements associated with these

- Users may download and print one copy of any publication from the public portal for the purpose of private study or research.

- You may not further distribute the material or use it for any profit-making activity or commercial gain

If the publication is distributed under the terms of Article $25 \mathrm{fa}$ of the Dutch Copyright Act, indicated by the "Taverne" license above, 


\title{
Spatio-temporal elements of articulation work in the achievement of repeat prescribing safety in UK general practice
}

\author{
Suzanne Grant, Jessica Mesman and Bruce Guthrie
}

\section{Introduction}

The use of medications that only clinically qualified professionals can prescribe is the most common technical intervention in healthcare. Prescriptions may be one-off to treat a shortlived illness, but three quarters of prescriptions and four-fifths of drug costs in the UK are accounted for by what is referred to in UK general practice as 'repeat prescribing' (Avery 2010). Repeat prescriptions are for chronically-used medications, typically authorised at the last medication review, and issued without a consultation between the patient and prescriber (Audit Commission 1994). Over recent decades, the proportion of items issued as repeat prescriptions has increased in parallel with the expansion in prescribing for long-term conditions in general practice. For example, a recent study by Petty et al. (2014) found that at least one repeat medication was prescribed to 43 per cent of the population at any one time, with this increasing to over 75 per cent of the population over 60 years of age.

Repeat prescribing is a high-volume process that involves multiple general practice professionals. UK general practices are typically general practitioner (GP)-owned businesses with $3,000-15,000$ patients, which will receive tens or hundreds of requests for repeat prescriptions for hundreds or sometimes thousands of different medications (many patients request multiple medications each time) each day, with a requirement to turn these requests into prescriptions for collection within 24-48 hours. UK general practices make significant use of clinical IT systems, which contain electronic patient records in the processing of repeat prescriptions to record, authorise, and issue repeat prescriptions. Although the signature of a qualified prescriber, usually a doctor, is legally required to issue a prescription, non-clinically trained administrative staff have responsibility for multiple tasks in the process.

Medication errors are a major cause of adverse events, with a recent study (Avery et al. 2013) stating that 4.9 per cent of repeat prescriptions contain an error. Given this, the measurement and management of prescribing is an important safety concern for the UK National Health Service (NHS) (Garfield et al. 2009, Taylor 1996). Research studies have also identified mild to moderate medication errors as relatively common and caused by a combination of individual factors (e.g. training, fatigue) and organisational factors (e.g. organisational culture, communication). For example, Avery et al. (2013) found that only 0.2 per cent of repeat prescription items in their study contained a severe error. While this research has been important for understanding the reasons why errors occur, it does not capture in

The Sociology of Healthcare Safety and Quality, First Edition. Edited by Davina Allen, Jeffrey Braithwaite, Jane Sandall and Justin Waring. Chapters (C) 2016 The Authors. Book Compilation (C) 2016 Foundation for the Sociology of Health \& Illness/Blackwell Publishing Ltd. 
detail the socio-cultural complexities of healthcare organisation and delivery, including differences in perceptions and definitions of risk and safety across organisational contexts and professional groups. In order to address these shortcomings, recent research on patient safety and quality improvement has emphasised the importance of organisational context and the complex power dynamics that operate therein on the way that risk is understood and quality and safety achieved (Iedema 2009, Macrae 2014, Rowley and Waring 2011, The Health Foundation 2014).

An important development within this emerging field of research has been the achievement of more nuanced understandings of the relationship between formal structural understandings and expressions of safety and informal everyday practice. In these studies, 'safety' is understood as a continuous enactment of the collective orientation of teams to each other, their resources, their physical environments, and their tasks (Iedema et al. 2014). One analytical lens that has been fruitfully applied within the context of secondary and community care has been Strauss's concept of 'articulation work' (Strauss 1985, 1988, 1993, Strauss et al. 1997). This concept has been used to examine the nature of collaborative activities between health and community care professionals, and the informal and often 'invisible' knowledge and resources that are necessary to conduct this work (e.g. Allen 2014, Hampson and Junior 2005, Postma et al. 2014, Timmermans and Freidin 2007). The concept of articulation work covers both formal routinized work and informal un/planned responses to un/anticipated contingencies (Allen 2014, Star 1991). Within the context of repeat prescribing, this work is dependent on the successful coordination of work between GPs and receptionists.

Receptionists have a central role in general practice work, with one of their key responsibilities being to act as administrative gatekeepers to GPs (Arber and Sawyer 1985, Ward and McMurray 2011). In the literature, the work of receptionists has often been contrasted with other members of the general practice team, in particular that of GPs (Copeman and Van Zwanenberg 1988). The majority of extant research on receptionists has focused on their role at the practice front desk and the extent to which they were understood and valued by patients (Hammond et al. 2013, Hewitt et al. 2009). Fewer studies have examined receptionists' relationship with other members of the practice team. Those studies that have examined this dynamic have tended to examine the ways that receptionists were valued by GPs, with Copeman and Van Zwanenberg (1988), for example, asking whether receptionists were 'poorly valued and underpaid?' An exception to this is a recent ethnographic study examining the repeat prescribing routines of four general practices in England by Swinglehurst et al. (2011) which showed that receptionists played a significant role in ensuring the quality and safety of repeat prescribing by making extensive use of tacit knowledge and situated judgements to bridge the gap between the formal organisational routine and the actual routine as it was played out in practice. However, given the central roles of both receptionists and GPs in this work and the potentially differing worldviews, logics, priorities and understandings of what constitutes safe, reliable care between these team members, it is equally important to develop a more detailed understanding of the nature of the coordination work that is undertaken between these professions and their relative contributions to repeat prescribing safety. Furthermore, given the cultural variation that exists across UK general practices as independent businesses (Grant et al. 2014), it will also be important to examine contextual differences between practices in greater detail across both formal and informal routines. This chapter ethnographically examines the formal and informal work employed by GPs and receptionists to safely conduct repeat prescribing work in UK general practice using Strauss's (1985) concept of 'articulation work' across a range of general practice contexts. 


\section{Methods}

The study was conducted during a Medical Research Council Population Health Scientist Fellowship held by SG (2009-13). The overall aim of the study was to examine the ways in which quality and safety were achieved across a range of organisational routines in UK general practice using ethnographic methods. This chapter focuses on the collaborative work required by general practice teams to achieve safety in their repeat prescribing routines.

\section{Sampling and data collection}

The study was conducted in NHS Scotland and NHS England from January 2011-April 2014 using a multi-site case study design across 8 urban and rural general practices (pseudonymised as 'Practice 1', 'Practice 2', 'Practice 3', etc.). Practices were purposively selected on the basis of their size (smaller with $\sim 4,000$ patients or larger with $\sim 9.000$ patients), socioeconomic deprivation of the population served (affluent, mixed or deprived), and location (urban and small town/rural) (Table 1). Data collection was in two phases, with an in-depth ethnographic study conducted in Practices 1-4 over a 24-month period in 2011/12, followed by more rapid ethnographic methods involving one week of fieldwork per practice applied in Practices 5-8 in 2013/14 focusing on specific organisational routines, including repeat prescribing.

A multi-sited ethnographic approach was adopted combining observation of everyday practice with interviews and documentary analysis. SG undertook 1,787 hours of ethnographic fieldwork from January 2011-April 2014. Informed consent was obtained from each practice team member prior to fieldwork commencing, and it was repeatedly explained that the researcher was interested in learning about the organisational culture, systems and processes of the practice and not in assessing individuals' performance. Fieldwork was undertaken with clinical, managerial and administrative staff during normal working hours in reception areas, back offices, consulting rooms, administrative offices, meeting rooms, coffee rooms and corridors. Detailed handwritten fieldnotes were made in full view of informants, and later transcribed for coding. Narratives were elicited from staff as they worked by the researcher asking them to talk through what they were doing and to 'think aloud' as they conducted their everyday work. These narratives were recorded with permission and later transcribed and coded. Documentary analysis of relevant written protocols (where available) and patient information leaflets from each practice was also conducted.

Towards the end of fieldwork in each practice, 62 semi-structured interviews were conducted by SG with GPs, practice nurses, practice managers and administrative staff (Table 2). Interviewees were selected based on observation of their work, and gave informed consent

Table 1 Practice characteristics and duration of fieldwork

\begin{tabular}{llllll}
\hline $\begin{array}{l}\text { Practice } \\
\text { no. }\end{array}$ & Country & $\begin{array}{l}\text { Practice } \\
\text { size }\end{array}$ & $\begin{array}{l}\text { Practice } \\
\text { urban/rural location }\end{array}$ & $\begin{array}{l}\text { Practice socioeconomic } \\
\text { deprivation }\end{array}$ & $\begin{array}{l}\text { Duration of } \\
\text { fieldwork }\end{array}$ \\
\hline 1 & Scotland & $\sim 4000$ & Urban & Mixed & In-depth \\
2 & Scotland & $\sim 9000$ & Urban & Deprived & In-depth \\
3 & Scotland & $\sim 5000$ & Urban & Mixed & In-depth \\
4 & Scotland & $\sim 8000$ & Rural & Affluent & In-depth \\
5 & England & $\sim 5000$ & Urban & Mixed & Rapid \\
6 & England & $\sim 6000$ & Rural & Affluent & Rapid \\
7 & Scotland & $\sim 9000$ & Urban & Deprived & Rapid \\
8 & Scotland & $\sim 8000$ & Rural & Affluent & Rapid \\
\hline
\end{tabular}


to participate before the interview commenced. The interview topics included: the interviewee's role within the practice; the practice organisational structure and culture; interviewee's descriptions of each organisational routine that they were involved in; the key ways in which they collaborated with other practice team members; and key strengths and weaknesses of each organisational routine in terms of patient safety. The interviews lasted 60 minutes on average, and were recorded and transcribed verbatim.

Table 2 Practice interviewees by profession

\begin{tabular}{lcclcc}
\hline Practice no. & GP & $\begin{array}{l}\text { Practice } \\
\text { nurse }\end{array}$ & $\begin{array}{l}\text { Practice } \\
\text { manager }\end{array}$ & $\begin{array}{l}\text { Administrative } \\
\text { staff }\end{array}$ & Total \\
\hline 1 & 2 & 2 & 1 & 3 & 8 \\
2 & 2 & 2 & 1 & 3 & 8 \\
3 & 2 & 2 & 1 & 2 & 7 \\
4 & 2 & 2 & 1 & 3 & 8 \\
5 & 2 & 1 & 1 & 3 & 7 \\
6 & 2 & 1 & 1 & 3 & 7 \\
7 & 2 & 2 & 1 & 2 & 10 \\
8 & 3 & 2 & 1 & 4 & 62 \\
Total & 17 & 14 & 8 & 23 & \\
\hline
\end{tabular}

\section{Analytical approach}

Analysis drew on the concept of articulation work, which Strauss (1985) developed to examine the nature of collaboration between professionals and the knowledge and resources that are necessary to conduct such work. Articulation work refers to 'the specifics of putting together tasks, task sequences, task clusters - even aligning larger units of work such as lines of work or subprojects - in the service of work flow' (Strauss 1988: 164). Since actors have to continuously adjust and realign their own individual actions in coordination with those of other actors, collaborative work requires the continuous readjustment of planned courses of action rather than a singular, linear course of action directed by formal rules and norms (Fjuk et al. 1997). Articulation work is the adjustment and alignment that is necessary to make organisational work happen, and individual activities cannot therefore be understood without taking into account the socio-cultural settings within which they take place.

Strauss (1993: 54) emphasises the temporal dimension of articulation work through the concept of 'trajectories', which are 'the course of any experienced phenomenon as it evolves over time' in ways that are not always predictable. Actors within a given trajectory have to establish and negotiate 'what work is to be done, to what standards, in what space, during what time period, with what resources, by whom, and with what payback' (Strauss 1993: 89). Strauss (1985) refers to the totality of tasks conducted over a period of time as an 'arc of work' which has a specific temporal trajectory, within which individuals have to constantly renegotiate their practices in the face of unexpected interactions and events. The trajectories of particular arcs of work are both multiple and intersecting and may be conducted sequentially, concurrently, or in phases, with specific practices being prioritised and structuring the execution of other coordinative activities (Fjuk et al. 1997, Strauss 1985). Strauss's concept of 'arc of work' resonated strongly with that of the repeat prescribing routine, which comprises a range of interconnected tasks conducted by different professionals over a specific period of time.

A further key feature of articulation work is the distinction that Strauss (1985) makes between formal and informal work where, in order to reduce the complexity of effort involved 
in articulation work, teams employ a range of mechanisms to standardise and simplify this work to render it predictable (e.g. standard operating procedures (SOPs) and protocols). Alongside this formal 'upper level' articulation work, organisational routines also contain informal elements that 'modif[y] action to accommodate unexpected contingencies' (Star 1991: 275) which are less well documented and/or officially authorised. Mesman (2010: 111) describes this as 'diagnostic work', which involves 'the recognition of the overall task structure, the ability 'to read' the conduct of co-participants and the identification of opportunities for interaction' in order for actors to 'act in the same present'. Individuals therefore need to be tuned into each other's tasks in order to recognise which actions should be adjusted, in what way, and when.

Finally, articulation work also has strong spatial dimension, which has been developed by Bardram and Bossen (2005) through the concept of 'mobility work'. Bardram and Bossen (2005: 136, italics in original) write: 'Mobility work designates the work needed to achieve the right configuration of people, resources, knowledge and place in order to carry out tasks at a certain point in time'. As a spatial complement to the temporally-focused SOPs, Bardram and Bossen (2005) developed the concept of 'standard operating configurations' (SOCs), which are 'a spatial setup fostering easy cooperation because of common knowledge and agreement as to use and navigation' (Bardram and Bossen 2005:138). Just like SOPs, SOCs provide a standard spatial arrangement of work practices.

Over the years, many studies of health and community work have proved the analytical strength of the concept of 'articulation work', particularly when applied in conjunction with a practice-based approach (Nicolini 2012) in which social phenomena are understood as being continually (re)created through active human agency (e.g. Allen 2014, Hampson and Junor 2005). For example, in her study of the invisible work of nurses, Allen (2014) employed a practice-based approach (Nicolini 2012) to distinguish between several forms of articulation work, including temporal, material, and integrative articulation. Others, like Fjuk et al. (1997), combined the work of Strauss with activity theory to examine the external factors and artefacts that influence actions. Our analysis underlines the added value of these and other theoretical elaborations to the concept of 'articulation work'.

Both fieldnotes and interviews were annotated with observational and theoretical notes and shared between the research team. This provided an opportunity for multidisciplinary reflection and enriched enquiry. The researcher (SG) read the interview transcripts to become familiar with the data. Preliminary themes were identified through scrutiny of initial transcripts and a coding framework was subsequently developed that was embedded in the data collected (Mays and Pope 1995), with Strauss's concept of articulation work used as a sensitising concept during the course of the analysis. This framework was consistently applied to the remaining transcripts using NVivo 8 (QSR International, Doncaster, Australia) software. The framework was refined according to emerging themes across the eight practices as the fieldwork developed. The modified framework was then reapplied to all transcripts, and this constant comparative method continued until no further categories emerged.

\section{Results}

Doctors and non-medical prescribers commonly issue prescriptions during face-to-face encounters, but most prescribing takes place without the patient being present, and starts with a request for a prescription made by phone, in writing, or at the reception desk. These requests can be for drugs that have been authorised for repeat issuing without a consultation ('repeats'), or for drugs which the patent has never had before, or drugs which the patient has 
had before but which have not been formally authorised for repeat issuing ('acutes'). As the demand for repeat prescriptions has increased over the past decades (Petty et al. 2014), practices have had to evolve these systems to manage this growing volume of work by balancing the rapid turnaround of prescription requests with the quality and safety of prescribing.

\section{Managing the growing volume of repeat prescribing}

Clinical professional control of prescribing relies on claims that this is the only way to ensure appropriate and safe use of potentially dangerous medicines, and is made concrete by the requirement for an individual qualified clinician (usually a GP) to sign each prescription and take responsibility for its issue. Key tasks in the repeat prescribing arc of work therefore included the management of requests and the printing of the correct prescriptions by receptionists, the delivery of a bundle of routine repeat prescriptions to GPs for signing with separate bundles of other requests to GPs to decide whether to issue, and the delivery of signed prescriptions or requests for further information or consultation back to patients. The volume of work involved was challenging for all eight practices to deliver:

It can be a huge bundle of stuff. You know, you could quite easily spend two or more hours doing repeat prescribing every day. Some days it's huge ... It usually breaks your spirits quite significantly. (Practice 4, GP2)

In the past, GPs often only signed repeat prescriptions for 'their' patients, but in all eight practices, all routine repeat prescriptions were signed by the daily 'duty doctor' in order to assure turnaround within 48 hours. However, the volume of prescriptions requiring signing made it impossible for the duty GP to double-check the details of every repeat prescription in the clinical record before signing them:

The vast majority are ones that are just routine and will be just signed. I mean when I say routine, obviously they have to be re-authorised every so often, but if they're authorised and it's all okay then it's usually just a question of signing it. (Practice 3, GP2)

Within the context of increased pressure on GPs' time, key requirements of the repeat prescribing system - careful checking of the accuracy and appropriateness of the request vs. rapid turnaround - compete for attention in a resource-constrained environment. GPs therefore relied on receptionists to appropriately issue and print prescriptions for signing with little further review, and to flag non-routine prescriptions for GPs' special attention. This socio-material configuration acted as a replacement for safety work that was fundamentally a GP clinical task, and required GPs to have to trust in the integrity of the repeat prescribing system and in the expertise of the receptionist:

You've just got to assume that the system is working. But it's a big task and it's ever-growing and there's no way of checking each and every script, you just can't do it. It's huge, I mean it's massive now. When I started 27 years ago it was a small task and there was a small bundle everyday but now it's probably 5 times as much as it was.

(Practice 4, GP1)

Three of the eight practices had no written protocol that specified how the 'system' was meant to work (Practices 1, 3 and 7) and in these practices, repeat prescribing was regarded as specialised work to be done by one full-time and long-serving expert receptionist in collaboration with the GPs, making a written protocol redundant (Table 3). The perceived safety 


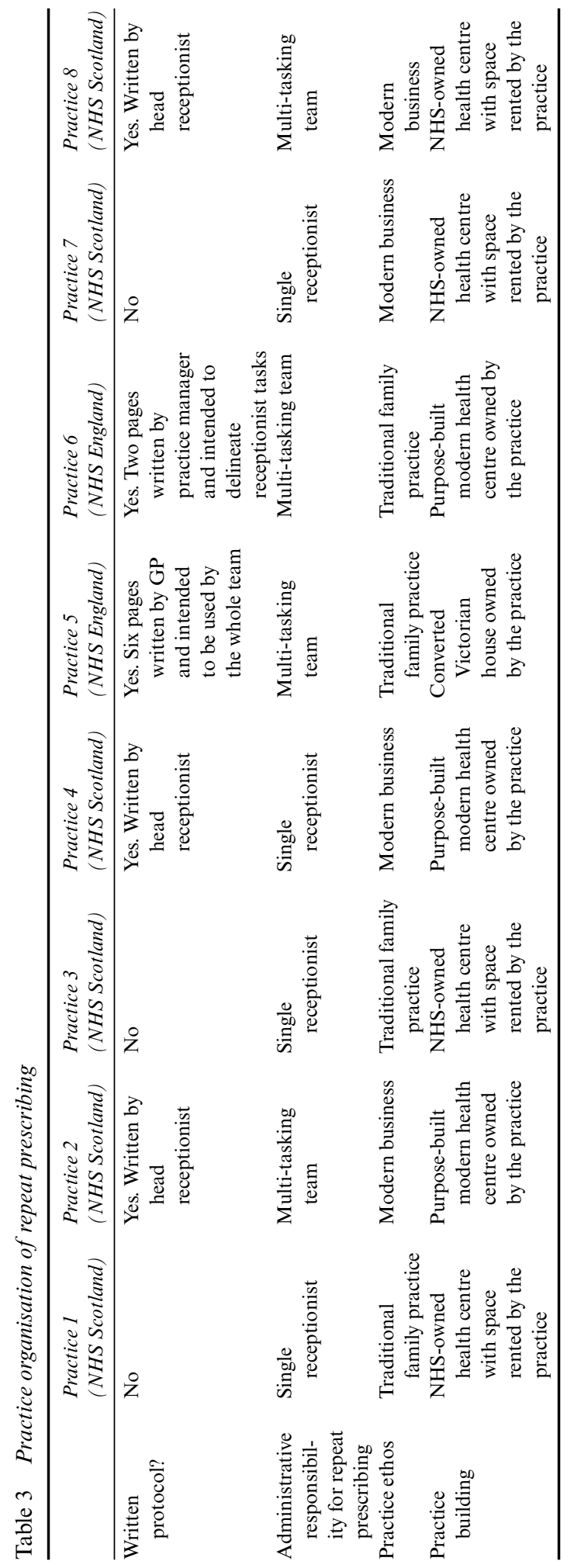


of this way of working lay in these experienced receptionists' accumulated knowledge of the clinical IT system, of drug names and doses, and of the 'regular' patients who were taking many medicines.

The remaining five practices had formal repeat prescribing protocols, written by one of the GPs (Practice 5), the practice manager (Practice 6), or the head receptionist (Practices 2, 4 and 8). These were primarily intended to be used by reception teams, who shared the repeat prescribing work to varying degrees. In Practice 4, repeat prescribing was also the responsibility of one receptionist, but in the other four practices with written protocols, it was a team-based task that practice managers had increasingly formalised as the volume of repeat prescribing had increased. In these four practices, repeat prescribing work was something that all members of the reception team were expected to have expertise in. Although it was acknowledged that there were safety risks involved in using less expert individuals and in the subsequent need for repeated handovers of complex information, this was considered less risky than relying on a single individual, which posed problems if that person was absent or left the practice.

Despite the close association between practices organising repeat prescribing work around teams and having written protocols, the protocols were not observed to be used during repeat prescribing work by anyone in the five practices, with many staff claiming never to have seen them or saying that they were part of their introductory pack but that they had never been read. Rather, GP and receptionist accounts of repeat prescribing work emphasised tacit knowledge as forming the basis of how repeat prescribing work was conducted on an everyday basis. Involved staff would readily provide detailed accounts of the whole arc of work, which included descriptions of the tasks that were required to be completed, the order that they should be done in, and the professionals who were involved in completing each task. In the practices that had them, protocols were largely used for the initial training of new staff, but had limited use beyond this.

While the same key elements of the arc of work were described by participants in all eight practices, practices varied in the order in which these elements were said to happen, and the combinations of staff involved at each stage. Initial interview descriptions also largely described what was intended to happen with routine prescription requests rather than how the whole range of requests were managed. Non-participant observation and think aloud interviews revealed a body of non-routine, 'invisible' articulation work (Allen 2014, Hampson and Junor 2005, Star 1991) conducted by GPs and receptionists to ensure delivery of the repeat prescribing arc of work. Space precludes a detailed description of all the work done, and so we focus on how requests were initially managed by receptionists in terms of those routinely issued versus those flagged for GPs' attention, how routine work was transferred from one member of the team to another, and how non-routine or urgent work was managed using a range of interruption practices.

\section{Initial management of requests by receptionists}

A core receptionist task in all of the practices was to distinguish between prescription requests that were authorised and legitimate 'repeats' that simply required GPs signing and prescriptions that required further attention from the duty GP, either because there were factors that made an authorised repeat problematic (for example, an early request), or because the request was for an acute prescription which was not authorised for regular issue. However, an important difference between the practices studied was in terms of the range of items that the GPs permitted the receptionists to issue and print.

In some practices, receptionists were only allowed to print items that were formally set up in the IT system as authorised 'repeats'. In Practices 4 and 6, receptionists were only allowed 
to issue and print items that were listed in the repeats section and so pre-authorised for regular issue, although the reasons given for this differed. Practice 6 was a rural dispensing practice (i.e. also took the pharmacists' role in dispensing drugs), and the GPs limited receptionist discretion since they perceived that not having an independent pharmacist involved in dispensing meant that any errors introduced earlier in the process were less likely to be detected. In Practice 4, the same tight control on what receptionists could issue was justified in the context of the GPs' pride in being a traditional family practice where GPs looked after 'their' patients. The GPs therefore dealt with all repeat prescription requests themselves during their shared morning coffee break, dividing the work between themselves based on who knew the patient best to both minimise error and provide personal care.

In the remaining practices, the receptionists were allowed to issue and print a limited range of 'acute' items beyond those formally authorised for repeat prescription. For example, the experienced specialist computer operator in Practice 1 had a list of acute drugs that she could issue attached to the wall, although these still required case-by-case checking by a GP, achieved by her attaching a yellow sticky note to the front of the printed prescription asking the duty GP if they were 'OK to give?'. While GPs acknowledged that this introduced some risk into the system since GPs could sign such a prescription without opening the record, the intent of this was to reduce the demands on GP time while ensuring that potentially problematic prescriptions were made obvious to the GP. In other practices, receptionists had fewer formal limits on what they could issue. In Practice 7, for example, the highly experienced 'repeats guru' was allowed to issue and print the widest range of medications of any practice, which was justified in terms of the experience that she had of both patients and the names and doses of different kinds of medication. She would thus frequently issue and print acute requests for GPs to sign and return to her without much oversight, which the practice felt was required to manage the very high volume of prescription requests made by the socioeconomically deprived population the practice served.

Across all of the practices, receptionists had to make decisions about how to direct GPs' attention to the problematic requests. Methods varied between practices and between individual receptionists in the same practice in terms of how this was done, with sticky notes, highlighter pens, ballpoint pens, notebooks and paperclips used to efficiently draw GPs' attention to elements of the request that the receptionist judged problematic. Receptionists devoted considerable time in all eight practices to this work, thus simplifying complex information to minimise the time GPs spent dealing with each problematic request.

In summary, timely management of the high volume of repeat prescribing requests in all practices required the delegation by GPs of key safety tasks to receptionists in order to save GP time, including safe identification of which drugs the patient was requesting, initial judgement about whether the request was an unproblematic repeat, and clear signalling to the GP of problematic requests. Practices varied in terms of what receptionists were permitted to do and how they simplified the following stage of work for GPs, but despite these differences, the repeat prescribing arc of work in all of these practices included large sets of tasks for the receptionists to undertake. Across each of the practices, receptionists played a key role in the simplification of the work of GPs and there was mutual coordination of each other's tasks across the repeat prescribing arc of work. The following section examines the transfer of prescriptions between GPs and receptionists in greater detail.

\section{Transferring practices for routine repeat prescribing work}

The efficient and effective transfer of tasks, documents and information between different members of the team was central to repeat prescribing work, but largely undocumented in the protocols that existed. A key role of the receptionists was to ensure that the 'pile' of 
standard repeat prescriptions was in the correct place at the correct time for the duty GP to collect and sign. Timing and positioning of the pile were crucial in order to constitute and maintain an arc of work to create a set of signed prescriptions for giving to the patient or pharmacist. The alignment of the different elements required both formal and informal articulation work with both temporal and spatial dimensions. Temporal articulation (Bardram 2000) refers to the articulation work that aims to position actions in the right moment and order. What is considered to be the right moment is based upon the prioritizing of different interdependent lines of work (e.g. seeing patients, doing house visits, the 48-hour turnaround of prescriptions). An important element in the temporal articulation work is the organising effect of deadlines. Here deadlines act as temporal markers within the overall flow of time. Rather than acting as a constraint, they act as a positive temporal beacon that one can focus on and align other activities around.

The transfer of prescriptions from the receptionist to the GP also required 'mobility work' (Bardram and Bossen 2005), with each practice having its own system for ensuring that this process took place smoothly and on time. For example, throughout the morning in Practice 2 , the receptionist would accumulate a pile of standard repeats in a wire tray on her desk, and ensure that by $2 \mathrm{pm}$ the pile was transferred to a purple folder in the duty doctor document tray for collection. In contrast, in Practice 1, the receptionist would continually bring prescriptions from her office to the table in the GPs' coffee room next door for the duty doctor to sign whenever they could throughout the day. The different approaches adopted by these practices constitute distinct modes of ordering (Law 1994). The receptionist in Practice 2 constituted part of a chain of intermediaries, with the trays and folders acting as a passageway that provided a trajectory for the prescription to travel from her desk to the GP. In other words, piles, trays and folders were 'coordinating artefacts' (Allen 2014: 61) that supported the arc of work. This consecutive mode of ordering differs from the one in Practice 1 where the mode of ordering was not compartmentalized but a continuous flow of work.

GPs were responsible for the timely return of signed repeats back to the receptionists, but how this was achieved varied between practices and between GPs within the same practice. In Practice 1, the duty GP would carry signed prescriptions back to the receptionist's office next door, whereas the duty GP in Practice 3 would hand them to the receptionist at the front desk who would then sort them into an alphabetically-arranged box for patients to collect. While the receptionists usually had strict deadlines to adhere to for their own cluster of tasks pre and post-GP signing, the GPs had greater autonomy and less pressure to be punctual, which then often required additional complex mobility work on the part of the receptionists to retrieve the prescriptions from the GPs. For example, in Practice 4 the duty GP was responsible for signing repeat prescriptions but if other work prevented them from doing this, prescriptions were shared equally between all of the GPs during coffee break to enable the receptionists 'to get the prescriptions moving' (Practice 4, Receptionist 2). However, this then created a retrieval problem for receptionists because it was unclear which GP had which scripts:

Some days you find they have shared them out and other days you've got them all back from the one person, you know? It isn't great for us as we've lost track of where everything has gone and we just have to keep going back to them and saying 'look these scripts haven't come back, we had them on Tuesday, this is by Thursday' you know ... You have to be assertive. (Practice 4, Receptionist 1)

In Practice 8, the receptionist would 'bundle [the scripts] out evenly' (Receptionist 1) across all of the GPs' trays at the back of reception so that they all 'got their quota'. While some GPs returned their morning prescriptions at lunchtime and then collected their next pile 
for signing, others returned prescriptions gradually between patients during their afternoon surgery creating delays for receptionists in making them available for collection.

In summary, this section has shown that even the most routine repeat prescribing work requires articulation work to ensure that it runs as smoothly and simply as possible. While the transfer of repeat prescription requests is a spatial task (the transfer of documents from A to $\mathrm{B}$ ) requiring mobility work by both receptionists and GPs, it is also highly dependent on temporal aspects of articulation work in order for this spatial articulation be achieved at the right moment. This temporal element has different implications for receptionists and GPs: while receptionists had fixed deadlines that acted as planning devices, GPs had greater temporal freedom, which partly related to their position of authority within the general practice organisation. The following section examines the least routine element of repeat prescribing, which is the role of interruptions in the achievement of this arc of work.

\section{Interrupting practices for non-routine repeat prescribing work}

The repeat prescribing arc of work is only one of many arcs of work happening simultaneously in all practices, including the opening, scanning and management of letters and test results, making appointments, and managing the constant flow of patients coming to the reception desk for multiple reasons. GPs and receptionists in all eight practices therefore regularly interrupted each other's work in order to complete their own work. The patient safety literature has tended to focus on the risks posed by interruptions, with distractions, for example, said to lead to transcription and other errors, or the non-completion of tasks. However, in all eight practices, interruptions were essential opportunities to efficiently interact with colleagues. The following fieldnote extract illustrates the kinds of interruptions that can take place for receptionists on a day-to-day basis:

09:31: Receptionist 1 brought another large handful of repeat prescription requests through to her office from the blue plastic box on the front desk of main reception area. This bundle was as large as the one that she had brought through earlier this morning when had she collected her first batch of requests from the box at 08:10. 'That's Monday mornings for you!' she exclaimed as she placed them down on her desk in front of her computer ... The rest of the repeat prescriptions pile from earlier that morning were still there, but she had decided to collect this more recent bundle whilst she was in the main reception asking the Duty Doctor to sign an urgent prescription request for a patient who had just been discharged from hospital.

09:34: Receptionist 1 took the first repeats request off the top of the pile and read through the key elements of the request ... She checked that the items were on repeats on the patient's record, that they were not over-ordering or due a medication review, and then authorised the request by printing off the prescription.

09:38: Just as she began working on [the next prescription request], Receptionist 4 came into the office and said 'Sorry to bother you, but Mr [name of patient]'s wife has just come in and handed in an urgent request for his pain killers - do you think you could just print it off now to save him waiting till tomorrow?' Receptionist 1 said she would as he was a 'poor soul' and placed the script that she had been working on to the left hand side of her keyboard with her pen on top to mark the place before printing off the prescription. Receptionist 4 thanked her and stuck her head out of the door to look down the corridor, saying that she would 'catch' GP2 and ask him to sign it right away as he was 'just going into the main reception to get his mail'. (Practice 1, fieldnotes, 14.09.12) 
Across all eight practices, the 'catching' of colleagues was necessary to ensure that all required work including repeat prescribing was done safely and effectively. On the basis of her ethnographic findings, Carroll (2009: 160) stresses that 'being available, being interrupted, interacting with colleagues and taking advantage of opportunistic communications, are fundamental to the progression of patient treatment and to redevelop effective daily work plans'. Although interruptions seem to be an indication of non-order or even chaos, Carroll writes that interruptions should not carry a solely negative connotation as they should be understood as a regular and characteristic feature of the work that also positively contribute to the flux of everyday work. Within the context of repeat prescribing, these non-ordered interactions allowed for adaptive decision-making regarding patients and work planning. There were many ways in which 'catching' could be done, including through electronic messaging, waiting for opportunities as clinical staff moved around the building, hovering in corridors or outside consulting rooms until someone was free, phoning them, or going to their workspace or consulting room. In other words, the permeability of time and space afforded order to the repeat prescribing routine and also enabled this order to be constantly updated (Carroll 2009: 161).

In some of the practices, the GPs actively encouraged the receptionist doing repeat prescribing to 'catch' them to discuss queries face-to-face because they perceived this to be more efficient. For example, in Practice 8, the GPs had deliberately placed the house visits book behind the repeats desk so that the receptionists had a continual flow of GPs available to 'catch' to complete non-standard work including repeat prescribing. The GPs also used this shared space to 'catch' receptionists to complete their own non-standard tasks such as phoning the hospital to resolve a query. Similarly, the shared coffee room in Practice 5 was situated behind the front office next to the repeat prescribing desk. This was intended to encourage the doctors to spend time in this area and facilitate informal and problem-solving interactions with nursing and reception staff, as the following fieldnote illustrates:

One of the young female salaried GPs comes into the reception area to collect her lunch from the fridge and make a cup of tea. She had been talking to one of the practice nurses sitting at the seating area about her forthcoming wedding plans. Receptionist 3 is at the repeats desk and asks her if she can ask her a 'quick question'. The GP comes across and the receptionist tells her that a female patient of $67 \mathrm{yrs}$ had just phoned in requesting morphine. The receptionist tells the GP that the patient 'sounded like she was in a lot of pain' and decided not to write it down in the black 'problem scripts' book, as the request wouldn't be dealt with until lunchtime. The receptionist asks what she should do. The GP tells her 'We don't normally do this, but this patient is elderly and in lots of pain, so I think it's OK to fax the morphine prescription to the pharmacist'. (Fieldnotes, Practice 5, 09.07.13)

For more urgent requests, receptionists would more actively seek to locate any or a particular GP. This was variously referred to as 'hovering' or 'lurking' around the practice corridors until they 'caught' a suitable doctor that could respond to their request. Iedema et al (2006) explain how this 'corridor work' turns a marginal space such as the corridor into a site of intense productivity that is an important resource for healthcare teams:

This is a unique site where final decisions can be held in abeyance and where uncertainties and provisional decisions can co-exist; a space where the fixities of hierarchy and specialization can be attenuated if not suspended, and a space where people can agree to work around rules and regulations; in short, a space where tasks and 
positionings become sufficiently provisional, flexible and negotiable to enable clinicians to weave the complexity of emerging facets of clinical practice into a workable and productive unfolding. (Iedema et al. 2006: 238)

Partly to reduce the risks associated with interruptions, the repeats receptionists in Practices 1 and 7 worked in specially-designated individual offices. This required them to employ different tactics to catch clinical staff for non-standard repeat prescribing work. In Practice 1, for example, the repeats receptionist would keep all problem scripts in a small pile to the side of her desk and 'catch' GPs or nurses opportunistically as they entered the office. However, if she required urgent advice or a signature, she would often 'keep an eye on the window' of the office that faced the main corridor to the GPs' consulting rooms and 'catch' a GP as they walked past. Across all of the practices, receptionists would regularly check the practice appointment system on their computer screen to see which GP was free at that moment, with many deliberately targeting specific GPs that they knew were more amenable to interruptions of particular types of request:

The girls at reception, we know which doctors would sign an urgent script, you know, without grumbling. So we kind of watch the screen to see who's going to be the next one up and then go down the corridor to catch them. If we have an urgent prescription that comes through, then we go tap on the door of a doctor and just say 'Please'. (Practice 8, Receptionist 1)

Some practices had created defined time for lower urgency catching, for example through having a number of 2-minute long appointment slots in the morning and afternoon for GPs to examine any 'problem queries'. This reduced interruption of GPs, but also meant that receptionists knew when particular tasks were due to be completed.

In other practices, the building layout mitigated against informal interaction, with Practices 2 and 4 for example having a separate GP corridor that was located at the opposite end of the practice from the reception. In these practices where there were fewer opportunities for face-to-face catching, there was a greater reliance by receptionists on electronic messaging and telephone calls, notably in Practices 2, 4, 6 and 7. Electronic messaging in particular had been actively developed in all four of these practices in order to create a clear audit trail for tasks being transferred (e.g. receptionists had to initial each communication for audit purposes) and to minimise time spent physically locating one another. The GPs in Practice 4, for example, described themselves as being at the cutting edge of technology, and receptionists made extensive use of instant messaging 'bubbles' which appeared instantly in the middle of the recipient's screen and facilitated rapid responses to simple queries. For example:

Receptionist 3 sends a 'bubble' [a message on the practice IT system that instantly appears in the middle of the recipient's screen] to GP2 saying '[first name of GP], pt forgot to ask for an rx for Priadel $200 \mathrm{mg}$. Could you write an rx and send to chemist? Thanks [initial of receptionist]'. GP2 responds several minutes later with: 'Do not issue. Make an appointment with patient to see me [initials of GP]'. The receptionist then phoned the patient to ask him to make an appointment with the doctor to discuss their request further. (Practice 4 , fieldnotes, 15.02.12; $\mathrm{pt}=$ patient, $\mathrm{rx}=$ prescription)

In summary, while interruptions have frequently been presented in the patient safety literature as risky activities that are conducive to errors, this section has shown that interrupting 
the routine flow of tasks in an arc of work was frequently employed by GPs and receptionists as a way to prioritise and manage urgent, higher-risk work.

\section{Discussion}

The application of Strauss's (1985) concept of articulation work to examine the formal and informal work required to safely conduct repeat prescribing work in UK general practice has revealed the central role of informal spatio-temporal re-adjustment and re-alignment by GPs and receptionists as positive, proactive safety solutions to continually relocate vulnerability in time and space. Through the lens of articulation work and the application of ethnographic methods, this study has also revealed the central role of the informal, invisible practices of general practice receptionists, with informal, cross-hierarchical communication often more effective than formal structures of communication. This discussion examines these issues in greater detail.

The application of articulation work to examine medication safety in UK general practice has enabled the exploration of the relationship between the formal, visible elements of repeat prescribing work alongside more informal, invisible elements, and their respective roles in the achievement of repeat prescribing safety. While some of the general practices in this study had written protocols that outlined a simplified version of the technical aspects of the process, the complexity of the work involved in coordinating repeat prescribing alongside the other arcs of work that were taking place concurrently (e.g. test ordering, patient consultations, opening mail) rendered such formal documents inadequate as practical navigational guides for GPs or receptionists. As recent studies have shown (Grol et al. 2008, Iedema 2009, Mesman 2009, 2010, 2011, Rowley and Waring 2011, Macrae 2014), the application of rules, guidelines, regulations and protocols for patient safety improvement will never fully eradicate the imperfect and contingent nature of everyday work practices and the presence of risk and vulnerability therein. The analytical lens of articulation work and the adoption of a practice-based approach (Nicolini 2012) has illuminated not just the risks, vulnerabilities and potential errors inherent in repeat prescribing work (e.g. transcription errors, communication errors), but also the local, informal resources of resilience and strength that were employed by practice team members in the achievement of repeat prescribing safety. Space was therefore both a challenge (invisibility when receptionists were located in separate rooms) as well as a solution (corridor work). Time was also both a challenge (shortage of time for GPs to conduct every element of the high-volume, safety-critical administrative work) as well as a solution (interruptions). By making explicit the mundane, local routines across the eight practices, we were able to 'exnovate' (Mesman 2011) (i.e. make visible) the already existing local competencies that were routinely employed but invisible to both rationalised models of organisational analysis (Star and Strauss 1999) and team members themselves because they were used every day. An analytical focus on visible and invisible work proved to be a powerful tool for developing a more nuanced understanding of both the vulnerabilities and strengths of repeat prescribing configurations across a range of organisational contexts (Hampson and Junor 2005).

This study was conducted across eight general practices in Scotland and England, which enabled a comparative examination of the strengths and vulnerabilities of different repeat prescribing routines. While the formal stages of the repeat prescribing routine were broadly similar across the eight practices, each had adopted different context-specific solutions that spanned organisational (e.g. multi-tasking team vs. individual repeats 'gurus'), temporal (e.g. fixed deadlines vs. flexible timings), and spatial (e.g. placing prescriptions to be signed in a 
tray for GPs to collect vs. placing them directly in the coffee room) spheres of practice. This study showed that across the eight practices, the different spatio-temporal configurations that were employed illuminated different organisational and individual strengths, while at the same time displacing different vulnerabilities across space and time. Thus, while the presence of multi-tasking teams, for example, reduced the risks associated with concentrating receptionist expertise in the hands of one person (e.g. during holidays or if that person left), it also increased the risk of error associated with multiple handovers. While the uniqueness of individual practice systems may in part reflect the status of general practices as small, independent businesses owned by GP partnerships with unique socio-cultural traits (Grant et al. 2014), by examining the informal articulation work involved in repeat prescribing work, we were also able to develop a more nuanced understanding of the complex trade-offs that different practice teams made through the range of approaches that were adopted based on organisational strengths and weaknesses and the application of tacit knowledge by key individuals across this arc of work (The Health Foundation 2014).

Alongside the complexities of coordinating the individual tasks within an arc of work, Strauss (1985: 9) highlights the nature of the interrelationship between the multiple arcs of work that take place simultaneously within an organisational setting, with 'one receiving more organisational priority than another at a given phase'. Within the patient safety literature, such intersections are frequently reported as challenges to patient safety and a key cause of error (e.g. Avery et al. 2013). However, in this study we found that these intersections were purposively employed by GPs and receptionists as opportunities for the achievement and maintenance of repeat prescribing safety in the face of an increasingly challenging workload. The completion of urgent work frequently resulted in the creation of impromptu spatial configurations across the practices beyond the SOCs that were typically employed by practices. Thus, interruptions provided informal, impromptu opportunities for GPs and receptionists to communicate and prioritise tasks in multi-purpose, 'liminal' (Iedema et al. 2006) spaces such as corridors, coffee rooms and back offices, and so prevent the arc of work from stalling. Similarly, deadlines were not simply engaged with as closures to particular tasks but rather as supportive structures for receptionists to coordinate the repeat prescribing arc of work whilst taking into account other arcs of work that interfered with its completion (see also Allen 2014). IT was also frequently employed by practices to crosscut spatial boundaries, particularly in practices where impromptu meeting opportunities were limited. These 'latent resources of safety' have been described by Mesman (2011) as taken-for-granted 'hidden competencies' that are held by individuals and teams. Time and space were therefore creatively, flexibly and positively employed by practices as tools of resilience rather than being fixed obstacles to be overcome.

Recent writers have emphasised that a key strength of articulation work has been in highlighting the informal, less visible elements of professional work (e.g. Hampson and Junor 2005, Postma et al. 2014, Star 1991). As was the case in Allen's (2014) study of UK secondary care, no practice team member was formally responsible for overseeing the repeat prescribing arc of work in any of the practices in this study. On a formal level, the GPs were responsible for authorising each prescription through signing them, and there were a range of intersecting hierarchical elements to the relationship between GPs and receptionists: receptionists were employees of the GP partnership, and it was the GPs who considered themselves to have 'overall authority' for the patients. Like nursing (Allen 2014), receptionist work is also highly gendered, with most receptionists being female and working class. While these underlying hierarchical features were present across all of the practices, this ethnographic study has also revealed the dynamic nature of the role boundary between GPs and receptionists, which varied depending on the nature of articulation work being conducted. 
While GPs' formal authority for repeat prescribing was physically enacted through their signing of each repeat prescription, it was the receptionists who were responsible for the initiation and overall coordination of the repeat prescribing arc of work. Gherardi (2006) writes that arcs of work are multiple and intersecting and with differing degrees of complexity or 'density'. Coordinating the overall navigation of multiple intersecting tasks with varying degrees of complexity required a great deal of creativity, flexibility and initiative on the part of receptionists in order to achieve the correct configuration of team members and artefacts in the right place at the right time (Allen 2014, Hampson and Junor 2005, Suchman 1987). The high volume of repeat prescription requests and the urgent nature of many of these requests meant that GPs placed a great deal of trust and authority in receptionists to ensure that prescriptions were filtered correctly. For example, receptionists had the authority to re-authorise repeat medication and authorise certain 'acute' requests in practices where the repeats volume was particularly high. Allen (2014:4) writes that invisible work lies 'at the heart of politics about what will count as work'. An examination of repeat prescribing invisible work has shown that receptionists were a key locus of strength in the repeat prescribing routine across all of the practices. However, due to the mundane, 'routine' and most importantly invisible nature of this work, it is rarely acknowledged and infrequently examined in depth. Building on Suchman's (2000) challenge to the dichotomy between 'knowledge' and 'routine' work, this study has therefore also highlighted the importance of tacit knowledge, practical reasoning and situational judgements on the part of administrative staff which are crucial for the maintenance of quality and safety in healthcare organisations.

\section{Conclusion}

This chapter has examined the different ways in which safety is achieved within a high-volume routine in UK general practice. As risky sociotechnical environments (Owen et al. 2009), healthcare organisations such as hospitals and general practices are inherently fallible, with technical and communication breakdowns, failures and incidents intrinsic characteristics of everyday work. The application of Strauss's (1985) concept of articulation work provided an effective analytical lens through which to examine the formal and informal ways in which repeat prescribing safety was achieved across eight UK general practice contexts and the strengths and vulnerabilities that exist across different practice socio-cultural contexts.

A recent report by The Health Foundation (2014) has highlighted the fundamental importance of understanding and taking into account internal organisational context in examining and implementing quality improvement work. In this report, (ibid.: 96) defines 'practical wisdom' (i.e. experience-based knowledge) and 'conjectural knowledge' (i.e. contextually-based stealth in achieving one's purpose) as essential elements of successful quality improvement work and evaluations. In this study, the application of ethnographic methods enabled an in-depth examination of each general practice context as well as the 'practical wisdom' and 'conjectural knowledge' employed by both GPs and receptionists in the achievement of prescribing safety. Of particular value has been the practice-based insight (Nicolini 2012) that articulation work provided into the informal, invisible elements of repeat prescribing work, including the positive role of receptionists and the value of informal over formal communication (Finn and Waring 2006) in the coordination of this work. Healthcare organisational research has focused mainly on the role of clinicians and the boundary between doctors and nurses (e.g. Allen 1997) or between clinicians and managers (e.g. Grant et al. 2015). With the exception of Swinglehurst et al. (2011), few studies have examined the role of receptionists in relation to the wider healthcare team in detail and on the role 
of context in augmenting this role. Given the central role of administrative team members in the quality and safety of healthcare delivery evidenced in this chapter, further research is required on the role of administrative staff within clinical contexts and on the nature of the clinical-administrative boundary as an addition to extant research on professional roles within organisational sociology.

The application of ethnographic methods has enabled us to 'exnovate' (Mesman 2012) the 'practical wisdom' and 'conjectural knowledge' (The Health Foundation 2014) employed by GPs and receptionists to conduct high-volume, everyday routine work safely and effectively. Further ethnographic studies are also required that employ the analytical lens of articulation work in order to better understand how safety and risk are understood in context. Alongside this, the study of informal, invisible safety practices can also form the basis of further 'exnovative' safety improvement work that employs ethnography and improvement methodologies such as video-reflexivity (Iedema et al. 2014) to foster further innovation and improvement from within healthcare organisations in order for healthcare teams themselves to better understand and build on their vulnerabilities and their strengths.

\section{Acknowledgements}

Suzanne Grant would like to thank all eight practices who generously gave up their time to participate in this study and from whom she learned so much. The authors also wish to thanks the editors and three anonymous reviewers for their constructive and insightful comments and suggestions, which greatly contributed to improving earlier versions of the manuscript. Suzanne Grant was funded by a Medical Research Council (MRC) Population Health Scientist Fellowship Award (G0802406).

\section{References}

Allen, D.A. (1997) The nursing-medical boundary: a negotiated order? Sociology of Health \& Illness, $19,4,498-520$.

Allen, D.A. (2014) The Invisible Work of Nurses: Hospitals, Organisation and Healthcare. New York: Routledge.

Arber, S. and Sawyer, L. (1985) The role of the receptionist in general practice: a 'dragon behind the desk?, Social Science and Medicine, 20, 9, 911-21.

Audit Commission (1994) A Prescription for Improvement: Towards More Rational Prescribing in General Practice. London: Stationery Office.

Avery, T. (2010) Avoidable prescribing errors: incidence and the causes, Prescriber, 21, 1, 52-5.

Avery, A.J., Ghaleb, M., Barber, N., Dean Franklin, B., Armstrong, S.J., Serumaga, B., Dhillon, S., Freyer, A., Howard, R., Talabi, O. and Mehta, R.L. (2013) The prevalence and nature of prescribing and monitoring errors in UK general practice - a retrospective case note review, British Journal of General Practice, 63, 613, 543-53.

Bardram, J.E. (2000) Temporal coordination of time and coordination of collaborative activities at a surgical department, Computer Supported Cooperative Work, 9, 2, 157-87.

Bardram, J.E. and Bossen, C. (2005) Mobility work: the spatial dimension of collaboration in a hospital, Computer Supported Cooperative Work, 14, 2, 131-60.

Carroll, K. (2009) Unpredictable predictables: complexity theory and the construction of order in intensive care. PhD dissertation, University of Technology, Sydney (UTS)

Copeman, J. and van Zwanenberg, T. (1988) Practice receptionists: poorly paid and taken for granted? Journal of the Royal College of General Practitioners, 38, 1, 14-16.

Finn, R. and Waring, J. (2006) Organisational barriers to architectural knowledge and teamwork in the operating theatre, Public Money and Management, 26, 2, 117-24. 
Fjuk, A., Smørdal, O. and Nurminen, M. (1997) Taking articulation work seriously - an activity theoretical approach. Unpublished paper submitted to ECSCW ‘97, Department of Informatics, University of Oslo.

Garfield, S., Barber, N., Walley, P., Willson, A. and Eliasson, L. (2009) Quality of medication use in primary care - mapping the problem, working to a solution: a systematic review of the literature, BMC Medicine, 7, 50.

Gherardi, S. (2006) Organizational Knowledge. The Texture of Workplace Learning. Oxford: Blackwell.

Grant, S., Guthrie, B., Entwistle, V.A. and Williams, B. (2014) A meta-ethnography of organisational culture in primary care medical practice, Journal of Health Organization and Management, 28, 1, $28-40$.

Grant, S., Ring, A., Gabbay, M., Guthrie, B., McLean, G., Mair, F., Watt, G., Heaney, D. and O'Donnell, C. (2015) Soft governance, restratification and the 2004 general medical services contract: the case of UK primary care organisations and general practice teams, Sociology of Health \& Illness, 37, $1,30-51$

Grol, R., Berwick, D. and Wensing, M. (2008) On the trail of quality and safety in health care, British Medical Journal, 336, 1, 74-6.

Hammond, J., Gravenhorst, K., Funnell, E., Beatty, S., Hibbert, D., Lamb, J., Burroughs, H., Kovandzic, M., Gabbay, M., Dowrick, C., Gask, L., Waheed, W. and Chew-Graham, C.A. (2013) Slaying the dragon myth: an ethnographic study of receptionists in UK general practice, British Journal of General Practice, 63, 608, e177-84.

Hampson, I. and Junor, A. (2005) Invisible work, invisible skills: interactive customer service as articulation work, New Technology, Work and Employment, 20, 2, 166-81.

Hewitt, H., McCloughan, L. and McKinstry, B. (2009) Front desk talk: discourse analysis of receptionist-patient interaction, British Journal of General Practice, 59, 565, 260-6.

Iedema, R. (2009) New approaches to researching patient safety, Social Science and Medicine, 69, 12, $1701-4$.

Iedema, R., Long, D., Carroll, K., Stenglin, M. and Braithwaite, J. (2006) Corridor work: how liminal space becomes a resource for handling complexities of multi-disciplinary health care, Pacific Researchers in Organizational Studies 11th International Colloquium, 238-47. http://search. informit.com.au/documentSummary; $\mathrm{dn}=305691933675194$;res=IELBUS, Accessed 27th July 2015.

Iedema, R., Mesman, J. and Carroll, K. (2014) Visualising Healthcare Practice Improvement: Innovation from Within. London: Radcliffe Publishing.

Law, J. (1994) Organizing Modernity. Oxford: Blackwell.

Macrae, C. (2014) Close Calls: Managing Risk and Resilience in Airline Flight Safety. Basingstoke: Palgrave.

Mays, N. and Pope, C. (1995) Rigour in qualitative research, British Medical Journal, 311, 6997, 109-12.

Mesman, J. (2009) The geography of patient safety: A topical analysis of sterility, Social Science and Medicine, 69, 12, 1705-12.

Mesman, J. (2010) Diagnostic work in collaborative practices in neonatal care. In Büscher, M., Goodwin, D. and Mesman, J. (eds) Ethnographies of Diagnostic Work: Dimensions of Transformative Practice. Basingstoke: Palgrave Macmillan.

Mesman, J. (2011) Resources of strength: An exnovation of hidden competences to preserve patient safety. In Rowley, E. and Waring, J. (eds) A Socio-cultural Perspective on Patient Safety. Aldershot: Ashgate.

Nicolini, D. (2012) Practice Theory, Work and Organization: An Introduction. Oxford: Oxford University Press.

Owen, C., Béguin, P. and Wackers, G. (2009) Risky Work Environments: Reappraising Human Work within Fallible Systems. Aldershot: Ashgate.

Petty, R., Zermansky, A.G. and Alldred, D.P. (2014) The scale of repeat prescribing - time for an update, BMC Health Services Research, 14, 76.

Postma, J., Oldenhof, L. and Putters, K. (2014) Organized professionalism in healthcare: articulation work by neighbourhood nurses, Journal of Professions and Organization, 2, 1, 61-77.

Rowley, E. and Waring, J. (2011) A Socio-cultural Perspective on Patient Safety. Aldershot: Ashgate. 
Star, S.L. (1991) Invisible work and silenced dialogues in knowledge representation. In Ericksson, I.V., Kitchenham, B.A. and Tijdens, K.J. (eds) Women, Work and Computerization: Understanding and Overcoming Bias in Work and Education. Amsterdam: Elsevier.

Star, S.L. and Strauss, A. (1999) Layers of silence, arenas of voice: the ecology of visible and invisible work, Computer Supported Cooperative Work, 8, 1, 9-30.

Strauss, A. (1985) Work and the division of labour, The Sociological Quarterly, 26, 1, 1-19.

Strauss, A. (1988) The articulation of project work: An organizational process, Sociological Quarterly, $29,2,163-78$.

Strauss, A. (1993) Continual Permutations of Actions. New York: Aldine de Gruyter.

Strauss, A., Fagerhaugh, S., Suczek, B. and Wiener, C. (1997) The Social Organization of Medical Work. Chicago, IL: University of Chicago Press.

Suchman, L. (1987) Plans and Situated Action: The Problem of Human-Machine Communication. Cambridge: Cambridge University Press.

Suchman, L. (2000) Making a case: knowledge and routine work in document production. In Luff, P., Hindmarsh, J. and Heath, C. (eds) Workplace Studies: Recovering Work Practice and Informing System Design. Cambridge: Cambridge University Press.

Swinglehurst, D., Greenhalgh, T., Russell, J. and Myall, M. (2011) Receptionist input to quality and safety in repeat prescribing in UK general practice: ethnographic case study, British Medical Journal, $343, \mathrm{~d} 6788$.

Taylor, R.J. (1996) Repeat prescribing - still our Achilles' heel?, British Journal of General Practice, 46, 412, 639-40.

The Health Foundation (2014) Perspectives on Context: A Selection of Essays Considering the Role of Context in Successful Quality Improvement. London: The Health Foundation.

Timmermans, S. and Freidin, B. (2007) Caretaking as articulation work: the effects of taking up responsibility for a child with asthma on labor force participation, Social Science and Medicine, 65, 7, 135163.

Ward, J. and McMurray, R. (2011) The unspoken work of GP receptionists: a re-examination of emotion management in primary care, Social Science and Medicine, 72, 10, 1583-7. 